\title{
The effect of microplastics on the growth of Paralichthys Olivaceus
}

\author{
Hui Lu${ }^{1,2}$, Liuqingqing Liu $^{1,2 *}$ \\ ${ }^{1}$ Institute of Coastal Environmental Pollution Control, Key Laboratory of Marine Environment and Ecology, Ministry of Education, \\ Ocean University of China, Qingdao 266100, China \\ ${ }^{2}$ College of Environmental Science and Engineering, Ocean University of China, Qingdao 266100, China
}

\begin{abstract}
In recent years, microplastics (MPs) pollution have become a global environmental issue, which aroused concern about their potential toxicity to marine organisms. However, due to the gradual depletion of natural resources, it had been rapidly developed in aquaculture. Therefore, this article studied the effect of polystyrene (PE) on the growth of Paralichthys Olivaceus. The surface of PE was rough and average size was $197.3 \pm 11.2 \mu \mathrm{m}$. The contact angle of PE was $100.3 \pm 2.5^{\circ}$, which indicated that PE had a hydrophobic surface. PE exposure had no significant effect on the body length growth of fish, but compared with CK treatment, the weight growth of fish in PE treatment group was slower, which increased by $36.3 \%$ on the 28 th day, but only increased by $10.9 \%$ in PE treatment group, indicating that PE could inhibit the growth of fish. PE led to the decrease of oxygen consumption rate of fish. On the 7th, 21st and 28th day, the oxygen consumption rate of fish decreased by $7.9 \%, 25.4 \%$ and $41.1 \%$, respectively. Based on the above results, we concluded that marine MPs could inhibit the growth of the benthic economic fish, which would disturb the balance of the marine ecosystem.
\end{abstract}

\section{Introduction}

With the wide use of plastic products, plastic waste pollution had aroused widespread concern. In 2019, the world plastic output was 368 million tons. There were many kinds of plastics, including PE, PS, PP, PVC, PET and so on. Plastic waste in the environment could be degraded into plastic with smaller particle sizes after a series of physical and chemical reactions. Since Thompson et al. defined microplastics as plastic particles with particle size less than $5 \mathrm{~mm}$ in 2004, MPs had been widely used to describe tiny plastic fragments in rivers, lakes and oceans [1]. Due to its small particle size, MPs were easily ingested by aquatic organisms and had toxic effects on the organisms. Studies had shown that MPs were easily ingested by plankton such as rotifers and daphnia, swimming organisms such as fish, and benthic organisms such as bivalves [2-5]. In addition to ingestion, MPs could also enter organisms through body surface attachment and respiration, produce toxic effects on aquatic organisms at the population, individual level, tissue and organ level, cell level, protein and gene level. In recent years, offshore aquaculture developed rapidly. Paralichthys Olivaceus had been rapidly developed in aquaculture because of its high economic value and gradual depletion of natural resources. Therefore, the benthic organism Paralichthys Olivaceus was easily affected by MPs exposure. Studies on the toxic effects of MPs on marine benthos showed that MPs could affect the feeding, movement and growth of marine benthos [6]. The smaller particle size, the easier it was for MPs to transport across the membrane to other tissues and accumulate, thus the greater the toxic effect on organisms [7]. The current research on the toxic effects of MPs on marine benthic organisms mainly focused on the toxic effects of smallsized commercial MPs on organisms, while under actual environmental conditions, the research on the biological effects of the dominant large-sized MPs was relatively scarce. Therefore, this study mainly studied the effect of the dominant large-sized MPs on the growth of marine benthic organism Paralichthys Olivaceus.

\section{Materials and methods}

\subsection{Material preparation and characterization}

PE debris was purchased from Shanghai Yangli Electromechanical Technology Co., Ltd. (Shanghai, China). The morphology of PE was observed by Confocal Laser Scanning Microscopy (A1, Nikon, Japan), and the particle size distribution of PE was statistically analyzed by Image $\mathrm{J}$ (http://imagej.net/). The functional groups of PE was analyzed by ATR-FTIR (Spectrum Two, PerkinElmer, UK). The hydrophilicity of PE was analyzed by Contact Angle Measuring Instrument (DSA20, KRUSS, Germany) [8].

\footnotetext{
${ }^{*}$ Corresponding author: liuliuqingqing $0630 @ 163 . c o m$
} 


\subsection{Exposure experiment}

The Paralichthys Olivaceus (length $9.06 \pm 0.24 \mathrm{~cm}$, weight $9.39 \pm 0.71 \mathrm{~g}$ ) was provided by Qingdao Haoruiyuan Aquaculture Co., Ltd. The purchased Paralichthys Olivaceus were randomly put into $25 \times 30 \times$ $30 \mathrm{~cm}$ glass tanks, containing $16 \mathrm{~L}$ of seawater and acclimated to the experimental conditions (temperature: $16 \pm 1{ }^{\circ} \mathrm{C}$; salinity: $32 \pm 1 \%$; pH: $8.4 \pm 0.1$; light: dark, $12 \mathrm{~h}: 12 \mathrm{~h})$ for 28 days. When the formal exposure experiment began, the domesticated Paralichthys Olivaceus were randomly assigned to the culture system. Ten fish were put into each tank, and two parallel was set in each treatment group. The exposure experiment was divided into two treatment groups, namely the blank control group (CK) and the PE treatment group (PE). The exposure method was water phase exposure, which was exposed for 28 days at the environmentally relevant concentration $\left(2 \mathrm{mg} \mathrm{L}^{-1}\right)$. On the 7th, 21st and 28th day after the exposure, four fish of Paralichthys Olivaceus (8 fish in each treatment group) were taken from each tank to measure the respiratory oxygen consumption rate. Then, the water on the surface of the fish was dried with absorbent paper, and the body weight and body length of the fish were measured. Finally, they were put back into the exposure system. The weight gain rate and oxygen consumption rate were calculated as follows [9]:

weight gain rate $=100 \times\left(\mathrm{W}_{\mathrm{t}}-\mathrm{W}_{0}\right) / \mathrm{W}_{0}$

Oxygen consumption rate $=100 \times\left(\mathrm{D}_{0}-\mathrm{D}_{\mathrm{t}}\right) /(\mathrm{W} \times \mathrm{t})$

where $\mathrm{W}_{0}$ was the initial weight of the fish $(\mathrm{g}), \mathrm{W}_{\mathrm{t}}$ was the final weight of the fish $(\mathrm{g}), \mathrm{D}_{0}$ was the initial dissolved oxygen of the fish $\left(\mathrm{mg} \mathrm{L}^{-1}\right), \mathrm{D}_{\mathrm{t}}$ was the final dissolved oxygen of the fish $\left(\mathrm{mg} \mathrm{L}^{-1}\right), \mathrm{W}$ was the body weight of fish (g); $\mathrm{t}$ was the measurement time (h).

\subsection{Statistical analysis}

All results were expressed as the mean values $(n=8)$. Error bars presented in the results represent the standard deviation. Significant differences between the treatments were analyzed by using one-way analysis of variance (ANOVA) with Duncan's multiple range test $(P=0.05)$ using Statistical Product and Service Solutions Software 20.0 (SPSS 20.0).

\section{Results and discussion}

\subsection{Morphology and particle size distribution of PE}

The morphology and particle size distribution of PE were showed in figure 1. The surface of PE was rough, with many folds and cracks. The particle size distribution was fitted by the gaussian curve, and the distribution characteristics showed normal distribution, the size of PE was mainly distributed in $100-300 \mu \mathrm{m}$, of which 180 $200 \mu \mathrm{m}$ accounted for $60 \%$, and $200-220 \mu \mathrm{m}$ accounted for $48 \%$. The average size of PE was $197.3 \pm 11.2 \mu \mathrm{m}$, which was the size of MPs in most seawater sampling surveys [10].

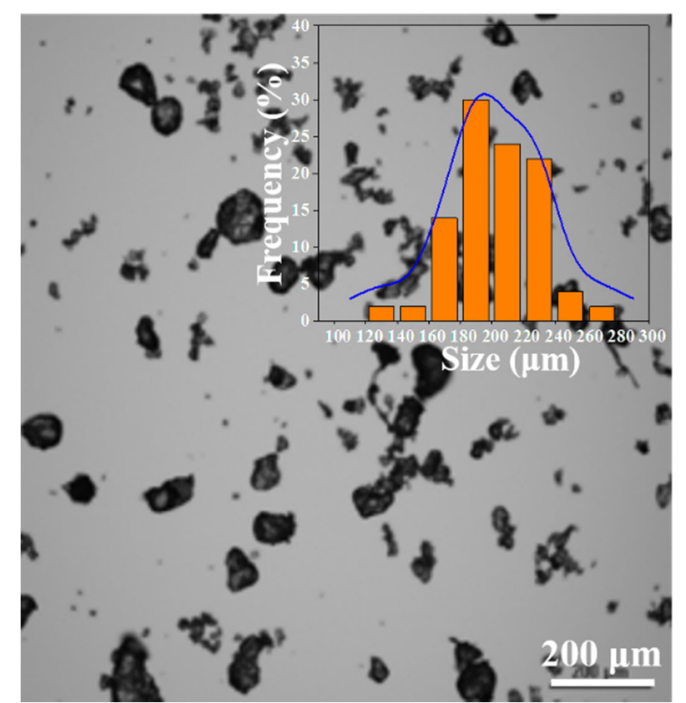

Figure 1. Morphology and particle size distribution of PE

\subsection{Surface chemical characteristics of PE}

The surface chemical characteristics of PE was showed in figure 2. ATR-FTIR spectrum showed analysis of functional groups on the surface of PE, it was found that when compared with standard PE, the MPs used in our experiment were indeed PE. In PE samples, $\mathrm{CH}_{2}$ stretching vibration absorption peak was at 1472 and 718 $\mathrm{cm}^{-1}$, aromatic C-H stretching vibration absorption peak was at 2915 and $2848 \mathrm{~cm}^{-1}$ [11]. These abundant alkanes fully demonstrate that PE was hydrophobic. This result was confirmed by the hydrophobic angle analysis, which showed that the contact angle of PE was $100.3 \pm 2.5^{\circ}$, and when the contact angle was $90-180^{\circ}$, the substance was hydrophobic. Therefore, such MPs may adsorbed heavy metals and organics, further harmed the ecological environment [12].

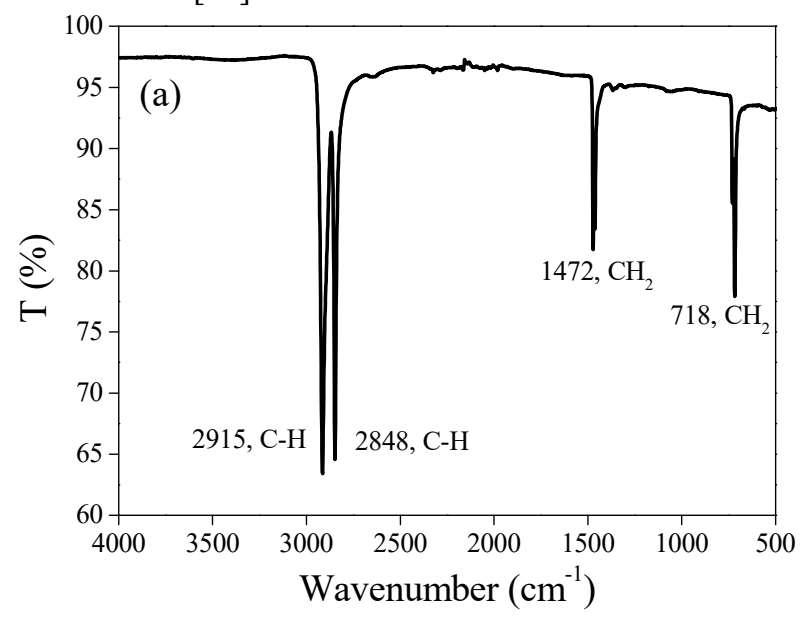




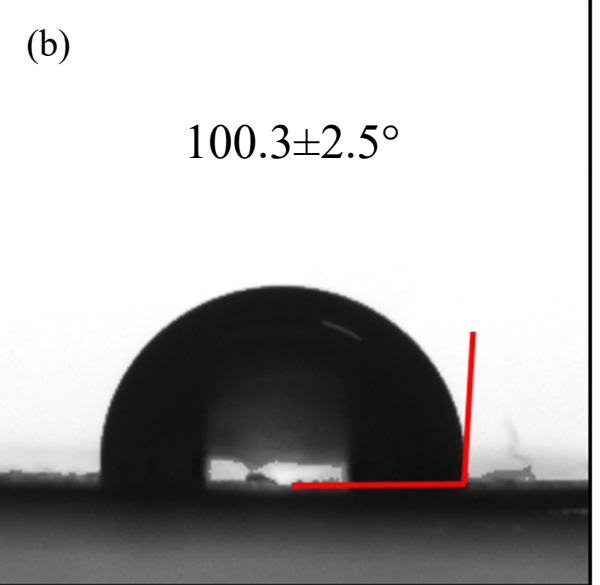

Figure 2. Surface functional groups (a) and hydrophobicity of PE (b)

\subsection{Effect of PE on the growth of Paralichthys Olivaceus}

The effects of PE on body length, body weight and weight gain rate of Paralichthys Olivaceus were showed in figure 3 . With the increase of exposure time, compared with 0 day, body length increased significantly at 28 day, at which CK treatment groups increased by $5.5 \%$, while PE treatment groups only increased by $2.8 \%$. In addition, compared with CK treatment groups, PE had no obvious effect on the body length growth of Paralichthys Olivaceus.

With the increase of exposure time, $\mathrm{CK}$ treatment groups had obvious effect on the body weight of Paralichthys Olivaceus. The Paralichthys Olivaceus in the CK treatment groups grew rapidly, while the PE treatment groups increased significantly after 21 day. It could be seen from the weight gain rate of Paralichthys Olivaceus that CK treatment groups increased 8.7\%, 12.0\% and $36.3 \%$ on the 7 th, 21 st and 28 th day, however, PE treatment groups only increased $2.6 \%, 4.5 \%$ and $10.9 \%$ on the 7th, 21st and 28th day. The results indicated that PE exposure could significantly reduce the growth rate of Paralichthys Olivaceus.

According to the above research, the exposure of PE could inhibit the growth of Paralichthys Olivaceus weight and reduce the health status of Paralichthys Olivaceus, which may be due to the accumulation of $\mathrm{PE}$ in the intestines, disrupting the metabolic balance and causing respiratory and metabolic disorders. The research of Chen et al. confirmed that exposure to MPs could reduce the thickness of the zebrafish's intestinal muscle layer [13]. This phenomenon was due to the thinning of muscle cells and loose connections, which would further affect the absorption of nutrients and ultimately put the zebrafish in an energy-depleted status. Therefore, the effect of oxygen consumption rate on the respiratory metabolism of Paralichthys Olivaceus was further explored.
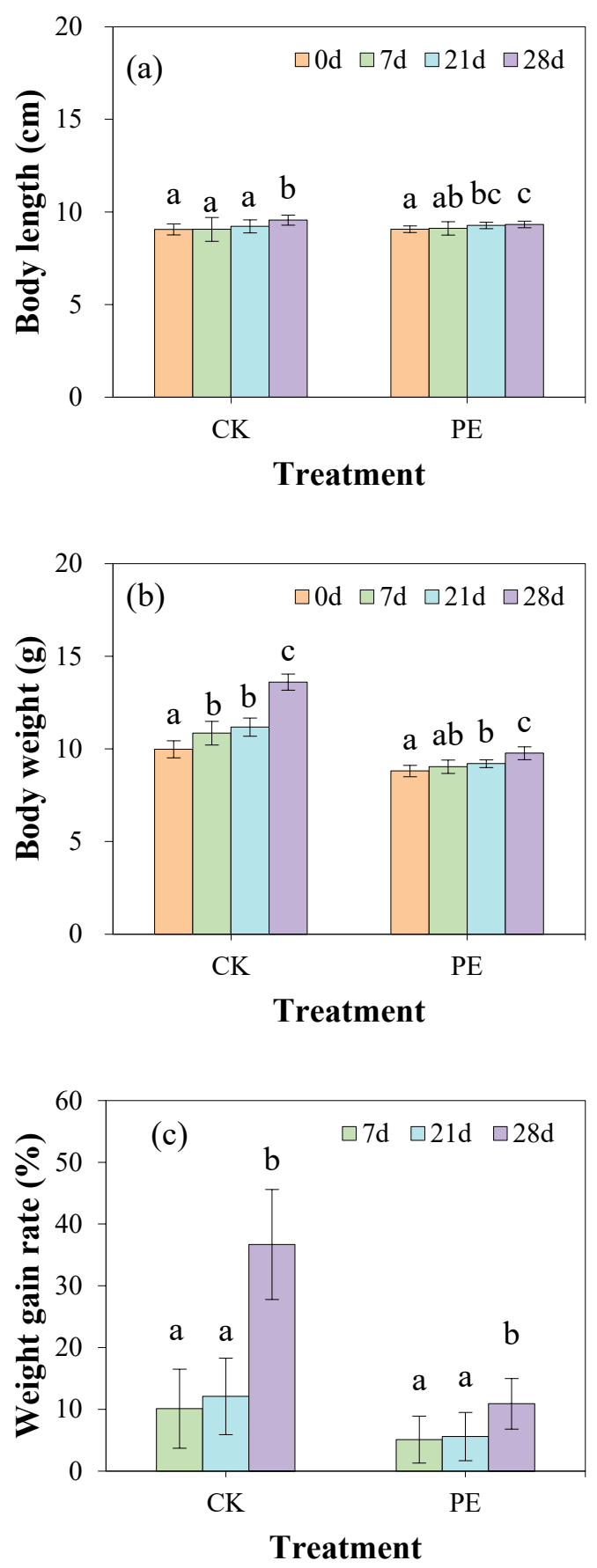

Figure 3. Effects of PE on body length (a), body weight (b) and weight gain rate (c) of Paralichthys Olivaceus. The bars represent standard deviation of the means $(n=8)$. The small letters on the bars indicate significant analysis, which were analysed by Duncan's test $(\mathrm{P}=0.05)$ using SPSS 20.0.

\subsection{Effect of PE on respiratory metabolism of Paralichthys Olivaceus}

The effects of PE on oxygen consumption rate of Paralichthys Olivaceus were showed in figure 4. It could be seen from the figure that the oxygen consumption rate of Paralichthys Olivaceus in CK treatment groups did not change significantly with the increase of time, but the oxygen consumption rate of Paralichthys Olivaceus in PE treatment groups decreased significantly, and decreased 
by $7.9 \%, 25.4 \%$ and $41.1 \%$ respectively on the 7 th, 21 st and 28th day. The results indicated that the exposure of PE could inhibit the respiratory rate of Paralichthys Olivaceus and disturb the respiratory metabolic balance of Paralichthys Olivaceus. However, previous studies found that compared with the $\mathrm{CK}$ groups, microplastics accumulated in mussels' gills after exposure to microplastics, so the oxygen consumption rate increased by $40 \%$, mainly because microplastics accumulated in organisms, which increased the oxygen demand of organisms [14]. In this study, the decrease of oxygen consumption rate of Paralichthys Olivaceus after exposure to microplastics may be due to the accumulation of microplastics in Paralichthys Olivaceus, which may further affect its growth and development.

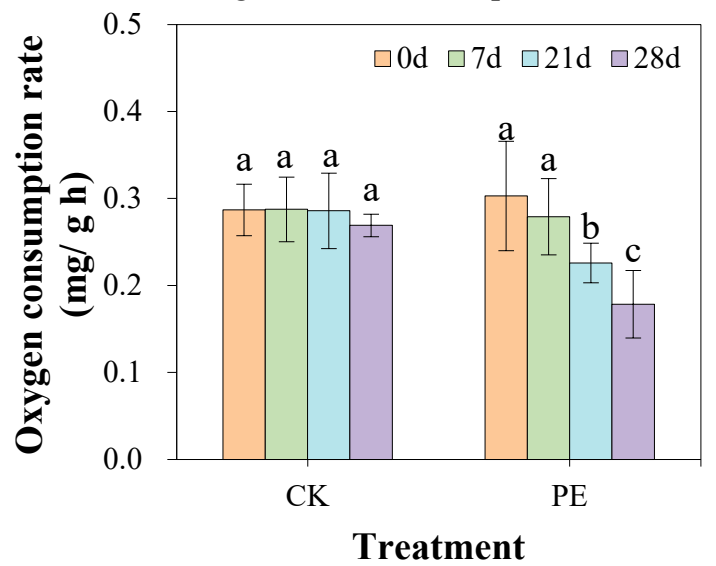

Figure 4. Effect of PE on oxygen consumption rate of Paralichthys Olivaceus. The bars represent standard deviation of the means $(\mathrm{n}=8)$. The small letters on the bars indicate significant analysis, which were analysed by Duncan's test ( $\mathrm{P}=$ 0.05) using SPSS 20.0.

\section{Conclusions}

The results showed that the exposure of PE could inhibit the respiration of Paralichthys Olivaceus and reduce its growth rate, which might be attached to $\mathrm{PE}$ and accumulated in gills, thus inhibiting its respiration. In addition, the exposure of PE had no obvious effect on the growth rate of Paralichthys Olivaceus, probably because PE were hydrophobic and suspended on the water surface, which was difficult to be captured by Paralichthys Olivaceus. Therefore, the potential risks of MPs in marine aquaculture should be paid enough attention, because it would affect the sustainable development of aquaculture and affect the ecological balance.

\section{Acknowledgments}

The research was supported by the joint fund of NSFC and Shandong Provincial Government, "Offshore Environmental Pollution, Effect and Control Theory and Method" (U1606404).

\section{References}

1. C.R. Thompson, Science 304, 838 (2004).

2. C. Gambardella, S. Morgana, S. Ferrando, M. Bramini, V. Piazza, E. Costa, F. Garaventa, M. Faimali, Ecotoxicol. Environ. Saf 145, 250-257 (2017).

3. E. Bergami, E. Bocci, M.L. Vannuccini, M. Monopoli, A. Salvati, K.A. Dawson, I. Corsi, Ecotoxicol. Environ. Saf 123, 18-25 (2016).

4. C.M Rochman, E. Hoh, T. Kurobe, S.J. Teh, Sci. Rep 3, 263 (2013).

5. M.A. Browne, A. Dissanayake, T. Galloway, D.M. Lowe, R.C. Thompson, Environ. Sci. Technol 42, 5026-5031 (2008).

6. R. Sussarellu, M. Suquet, Y. Thomas, C. Lambert, C. Fabioux, M.E.J. Pernet, A. Huvet, PNAS 113, 24302435 (2016).

7. Y. Lu, Y. Zhang, Y. Deng, W. Jiang, Y. Zhao, J. Geng, H. Ren, Environ. Sci. Technol 50, 4054-4060 (2016).

8. L.P. Luan, X. Wang, H. Zheng, L.Q.Q. Liu, X.X. Luo, F.M. Li, Mar. Pollut. Bull 139, 346-354 (2019).

9. X. Wang, H. Zheng, J. Zhao, X.X. Luo, Z.Y. Wang, B.S. Xing, Environ. Sci. Technol 54, 6202-6212 (2020).

10. Y. Cho, W.J. Shim, M. Jang, G.M. Han, S.H. Hong, Environ. Pollut 245, 1107-1116 (2019).

11. B. Gewert, M.M. Plassmann, M. MacLeod, Environ. Sci.: Processes Impacts 17, 1513-1521 (2015).

12. F. Yu, C.F. Yang, Z.L. Zhu, X.T. Bai, J. Ma, Sci. Total Environ 694, 133643 (2019).

13. Q.Q. Chen, C. Lackmann, W.Y. Wang, T.B. Seiler, H. Hollert, H.H. Shi, Aquat Toxicol 224, 105521 (2020).

14. A.F. Pedersen, K. Gopalakrishnan, A.G. Boegehold, N.J. Peraino, J.A Westrick, D.R. Kashian, Environ. Pollut 260, 113964 (2020). 